\title{
Microscopic models for direct inelastic scattering and direct preequilibrium emission: nucleon induced reactions
}

\author{
M. Dupuis ${ }^{1, a}$, E. Bauge ${ }^{1}$, L. Bonneau ${ }^{3}$, J.-P. Delaroche ${ }^{1}$, T. Kawano ${ }^{2}$, S. Karataglidis ${ }^{4}$, and S. Péru ${ }^{1}$ \\ 1 CEA, DAM, DIF, F-91297 Arpajon, France \\ 2 Theoretical Division, Los Alamos National Laboratory, Los Alamos, New Mexico 87545, USA \\ 3 Université Bordeaux 1, CNRS/IN2P3, Centre d'Etudes Nucléaires de Bordeaux-Gradignan, F-33175 Gradignan, France \\ 4 Department of Physics and Electronics, Rhodes University, Grahamstown 6140, South Africa
}

\begin{abstract}
We have developed microscopic models for nucleon induced inelastic scattering and one-step direct preequilibrium emission. These models are based on reliable effective in-medium two-body interactions and a microscopic description of the ground and excited states of target nuclei. No arbitrary renormalization process enters our analyzes and the predictions are directly compared to experimental data. The nuclear structure information are obtained in the Random Phase Approximation (RPA) framework with the Gogny force, which provides accurate descriptions of spherical nuclei without pairing. For medium energy (50-200 MeV) proton induced reactions, this approach gives very good predictions for direct inelastic scattering and for the first-step in direct preequilibrium emission. The one-step preequilibrium model has also been extended to fast neutron scattering (10-20 MeV) for the ${ }^{90} \mathrm{Zr}$ target described with RPA theory, and for axially deformed nuclei with a simpler description of the excited states (i.e. particle-hole excitations). Predictions of the reaction model reproduce well experimental data for ${ }^{90} \mathrm{Zr}$. For deformed targets $\left({ }^{232} \mathrm{Th}\right.$ and $\left.{ }^{238} \mathrm{U}\right)$, our calculations underestimate the data at high emission energy. The cross section missing for both actinides may stem from the excitation of vibrational states with excitation energies lower than $5 \mathrm{MeV}$ which are not described with incoherent particle-hole excitations. This defect might be cured if the target spectra are described within the Quasi-particle-RPA (QRPA) theory recently implemented with the Gogny force.
\end{abstract}

\section{Introduction}

The double-differential nucleon emission following medium energy nucleon induced reactions on a target in its ground state can be accounted for considering mainly three different reaction processes, namely direct process, which includes elastic scattering and inelastic scattering to discrete states, preequilibrium emission, and formation of and evaporation from a compound nucleus. One has also to consider evaporation from fission fragments if the target is a fissionable nucleus. Numerous phenomenological models exist to describe these processes. These models generally contain one or several parameters adjusted to reproduce experimental data. In contrast, microscopic models are under development to reduce the part of phenomenology in the treatment of all reaction mechanisms, from direct reactions to fission. In this work, we focus on progress in microscopic models for inelastic scattering to discrete states and preequilibrium emission in intermediate energy nucleon induced reactions.

We present quantum mechanical calculations of inelastic scattering and first order approximation to the preequilibrium multistep direct process (MSD), namely the onestep process [1]. Our model is based on: i) a reliable twobody effective force, to represent the residual NN interac-

\footnotetext{
a e-mail:marc.dupuis@cea.fr
}

tion between incident nucleon and target nucleons during an inelastic process, and ii) a microscopic description of the target states. For closed or near-closed-shell nuclei, the Self-Consistent Random Phase Approximation (SCRPA) with the Gogny D1S interaction [2] is used to obtain the structure information, including a good description of collective states which play an important role in both direct inelastic and preequilibrium reactions. For axially deformed nuclei, a Hartree-Fock+BCS model with a Skyrme interaction serves to define the structure information used in our one-step calculations. The present models for both inelastic scattering and preequilibrium emission do not contain any adjustable parameters, so the results can directly be compared with the data. A recent extension of the RPA theory to deformed nuclei (i.e. QRPA), as implemented with the Gogny force, provides spectroscopic information to high excitation energy. Results obtained for ${ }^{238} \mathrm{U}$ are briefly discussed. Such QRPA calculations will serve as a guide to improve reliability of present reaction models for deformed nuclei.

\section{Inelastic scattering to discrete states}

For inelastic scattering of a nucleon off a zero-spin ground state, differential cross sections have been calculated using

This is an Open Access article distributed under the terms of the Creative Commons Attribution-Noncommercial License, which permits unrestricted use, distribution, and reproduction in any noncommercial medium, provided the original work is properly cited. 
the distorted wave approximation (DWA) expression for the transition amplitude

$$
T=\left\langle\chi^{-}\left(\mathbf{k}_{f}\right), n\left|\hat{V}_{\text {eff }}\right| \chi^{+}\left(\mathbf{k}_{i}\right), \tilde{0}\right\rangle,
$$

associated with excitation $|n\rangle$ of the target originally in its ground state $|\tilde{0}\rangle$. The incoming $\chi^{+}\left(\mathbf{k}_{i}\right)$ and outgoing $\chi^{-}\left(\mathbf{k}_{f}\right)$ distorted waves were obtained by solving the onebody Schrödinger equation describing elastic scattering. That involved the non-local, $g$-folding optical potentials [4] formed using the target state structure (one body density matrix) described within the SCRPA/D1S framework for doubly-closed shell nuclei [3]. The effective interaction used in $g$-folding is the Melbourne $g$-matrix (solution of the Brueckner-Bethe-Goldstone equations [4]) which includes central, tensor and spin-orbit components that are all complex, energy and density dependent. The optical potential so formed is thus complex and energy dependent. The residual interaction $\hat{V}_{\text {eff }}$ for generating transitions, Eq. (1), is also the Melbourne $g$-matrix. In the RPA theory an excited state $|n\rangle=|N J \Pi M\rangle$ of multipolarity $J, \Pi$ and spin projection $M$, is written as a one quasi-boson excitation of the correlated ground state $|\tilde{0}\rangle$, namely

$$
\begin{aligned}
|n\rangle= & |N J \Pi M\rangle=\Theta_{N J \Pi M}^{+}|\tilde{0}\rangle \\
& =\sum_{p h \in(J \Pi)}\left[X_{p h}^{N} A_{J M \Pi}^{+}(p \tilde{h})-Y_{p h}^{N} A_{J \bar{M} \Pi}(p \tilde{h})\right]|\tilde{0}\rangle .
\end{aligned}
$$

The different quantities and operators in this equation are defined in [3]. Detailed description on the SCRPA calculations with Gogny force are given in [5]. We only remind that the operator $A^{+}(p \tilde{h})(A(p \tilde{h}))$ creates (annihilates) a particle-hole pair on the correlated ground state. Separate structure calculations have also been performed using a single particle-hole excitation on the uncorrelated HartreeFock (HF) ground state $|H F\rangle$

$$
|p h\rangle=A_{J M \Pi}^{+}(p \tilde{h})|H F\rangle, \quad|H F\rangle=\prod_{h} a_{h}^{+}|-\rangle,
$$

where $|-\rangle$ is the particle vacuum, to asses the role of collectivity in preequilibrium reactions (see Sec. 3).

The fully antisymmetric formulation of the transition amplitude with the different components of the Melbourne g-matrix involves different combinations of the one body density matrix elements which cannot simply reduce to local matter transition densities [6]. Thus the structure information that enters the DWA matrix elements are the full one body density matrices, namely all the $X$ and $Y$ components in Eq. (2).

We present differential cross sections calculated for ${ }^{208} \mathrm{~Pb},{ }^{116} \mathrm{Sn},{ }^{90} \mathrm{Zr},{ }^{40,48} \mathrm{Ca}$ and ${ }^{16} \mathrm{O}$. Comparisons with experimental data are shown in Fig.1(a,b) for proton inelastic scattering. Illustrations are displayed for incident energies in the range 54 to $201 \mathrm{MeV}$ leading to excitations of $2_{1}^{+}$, $6_{1}^{+}, 7_{1}^{-}, 8_{1}^{+}, 10_{1}^{+}$and $12_{1}^{+}$states in ${ }^{208} \mathrm{~Pb}$. The agreement between calculations and data ranges from good to excellent. For the $2_{1}^{+}$excited state, (a), cross sections are shown at six proton incident energies: our calculations agree very well with the experimental data, in shape and in magnitude. This comparison illustrates the quality of the Melbourne $g$-matrix (including its energy dependence) as well as that for the underlying SCRPA description of the $2_{1}^{+}$ state. Good agreement between calculated cross sections and data also is evident for the $6_{1}^{+}$to $12_{1}^{+}$excitations, (b), at selected incident energies in each case. The same microscopic model was used in [6] to analyze the excitations of $3_{1}^{-}, 4_{1}^{+}, 5_{1}^{-}$and $5_{2}^{-}$states and the predicted cross sections were also in very good agreement with the data. A minor disagreement is observed at angles above $30^{\circ}$ for the $6_{1}^{+}$ excitation and above $45^{\circ}$ for the $77_{1}^{-}$excitation where our predictions do not exactly match the data. However, firmer conclusions will require new inelastic scattering measurements for these two states $\left(6_{1}^{+}\right.$and $\left.7_{1}^{-}\right)$to further challenge our model predictions.

We depict in Fig.1(c) the same comparison extended to the first $3^{-}$excitation in the other doubly-closed-shell nuclei ${ }^{16} \mathrm{O}$ and ${ }^{40,48} \mathrm{Ca}$ as well as neutron or proton closedshell nuclei ${ }^{90} \mathrm{Zr}$ and ${ }^{116} \mathrm{Sn}$, respectively. The overall agreement between calculations and data is still very good. The good results obtained for ${ }^{90} \mathrm{Zr}$ and ${ }^{116} \mathrm{Sn}$ described in the RPA framework, which neglects the pairing correlations, is surprising especially for ${ }^{116} \mathrm{Sn}$ where these are strong. Thus, new studies based on the more appropriate QRPA theory should be performed to reach deeper understanding of these features.

\section{One-step direct preequilibrium emission from spherical targets}

The quantum preequilibrium model for MSD process is well established and details of the theory can be found in $[1,7]$. We are content to give the expression of the double-differential cross section corresponding to the onestep emission in the interval $\left[E_{k_{f}}-\Delta, E_{k_{f}}+\Delta\right]$

$$
\begin{aligned}
\frac{d^{2} \sigma\left(\mathbf{k}_{i}, \mathbf{k}_{f}\right)}{d \Omega_{f} d E_{k_{f}}}=\frac{1}{2 \Delta} \int_{E_{k_{f}}-\Delta}^{E_{k_{f}}+\Delta} d E \frac{\mu^{2}}{\left(2 \pi \hbar^{2}\right)^{2}} \frac{k_{f}}{k_{i}} \\
\sum_{F}\left|T_{F \leftarrow 0}^{(1)}\right|^{2} \delta\left(E_{k_{i}}-E-E_{F}\right),
\end{aligned}
$$

where $T_{F \leftarrow 0}^{(1)}$ is the first order transition amplitude. The sum over $F$ accounts for all the excitations in the target which satisfy energy conservation, and the amplitude $T_{F \leftarrow 0}^{(1)}$ corresponds to the DWA amplitude $T$, Eq. (1), for excitations in the continuum.

For proton scattering, we use the Melbourne g-matrix to built the optical potentials which provide the distorted waves and to calculate the transition amplitudes. We show in Fig. 2 comparisons between experimental data and onestep calculations with RPA solutions (full curves) for inelastic scattering of $120 \mathrm{MeV}$ protons off ${ }^{90} \mathrm{Zr}$. Figs.2(a,b) display the angular spectra of protons emitted at: (a) $\Theta=35^{\circ}$ for $20-120 \mathrm{MeV}$ and, (b) $\Theta=45^{\circ}$ for 100 $120 \mathrm{MeV}$. Above $80 \mathrm{MeV}$ of secondary energy, our predictions reproduce the data well. At lower energy calculations underestimate the data, which points to the need for 

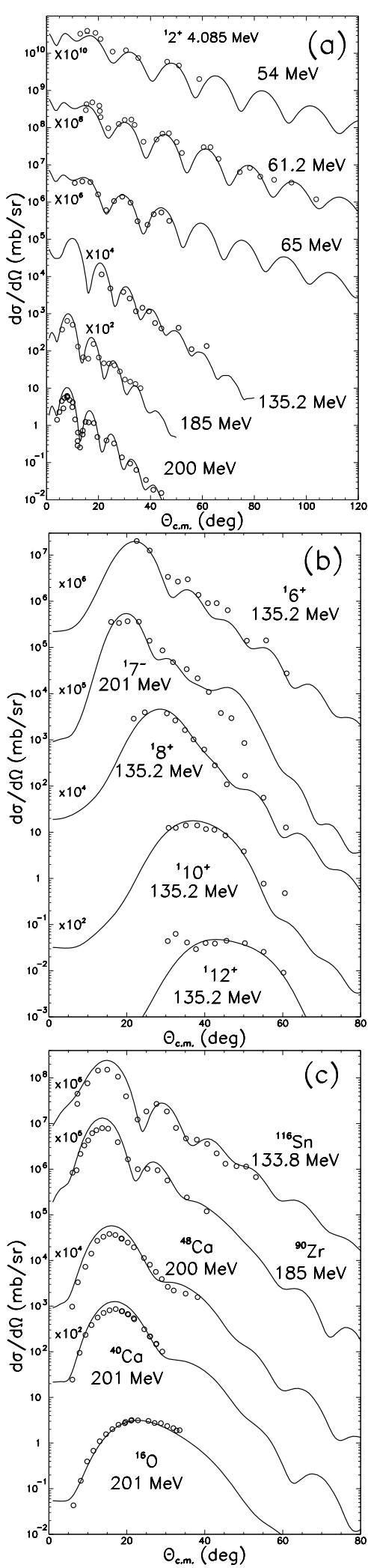

Fig. 1. Proton inelastic scattering leading to: $(a, b)$ the various excitations in ${ }^{208} \mathrm{~Pb}$, and (c): $3_{1}^{-}$excitations in other targets. Multipolarities, incident energies and targets are indicated on the plots. The results of the Melbourne+SCRPA/D1S model cross sections are displayed by solid curves. The open circles are the experimental data (see [6] for references).
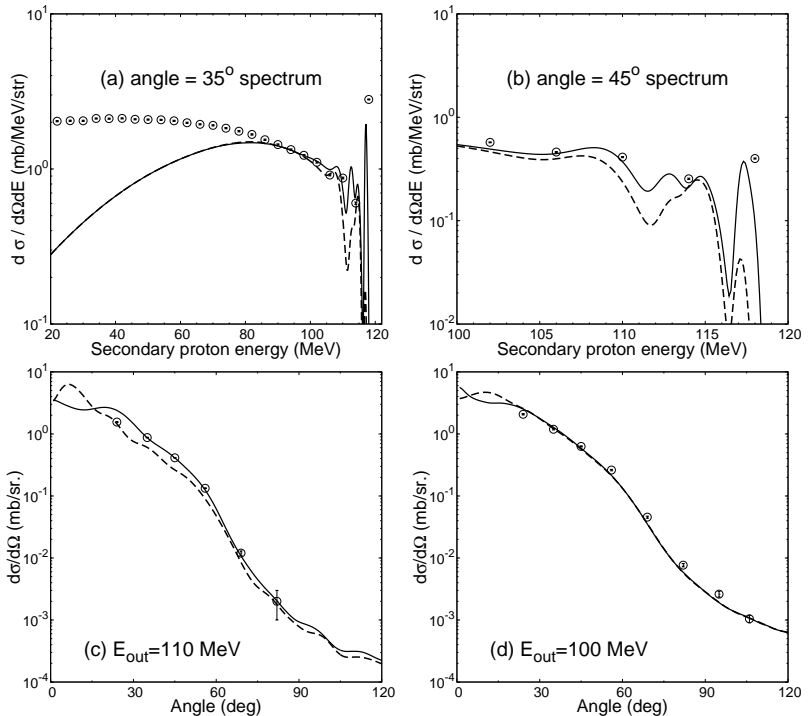

Fig. 2. Double-differential cross sections for inelastic proton scattering off ${ }^{90} \mathrm{Zr}$. Comparisons between experimental data [8] (open circles) and one-step calculations with RPA (full curves) or particle-hole (dashed curves) wave functions.

including second and higher order processes. In Figs.2(c) and (d) proton angular distributions are displayed at 110 and $100 \mathrm{MeV}$, respectively. Our calculations reproduce the data well at all angles. We note that at lower energy (not shown), the calculations underestimate data at backward angles since contributions from other reaction processes should be considered as well.

To emphasize the importance of a full RPA description of the target spectra, we performed the same preequilibrium calculation using particle-hole excitations of the HF/D1S ground state. With particle-hole excitations we underestimate the data for an emission energy above $105 \mathrm{MeV}$ and $\Theta \lesssim 70^{\circ}$ (dashed lines on Fig.2(b) and Fig.2(c), respectively). The differences between continuous and dashed curves come from the collectivity of the RPA excitations which gets lost in the particle-hole excitation picture. Indeed, for a specific excitation in the target, the inelastic cross section magnitude closely follows that for the reduced transition probability, which is much larger for collective excitations. This demonstrates that, at least, a full RPA description of all target states in closed shell nuclei is suitable to account well for the first step emission process.

For lower incident energies, in particular for 14-18 $\mathrm{MeV}$ neutrons, the above calculations are no longer valid because: i) the g-matrix interaction needs renormalization [9] which implies having recourse to phenomenological potentials, and ii) reactions processes other than one-step direct need be considered. Consequently, we will use the Koning-Delaroche potential [10] to generate the distorted waves. For the same reasons, using Melbourne g-matrix for the effective interaction in the transition amplitudes might not be appropriate. This is why we have tested other effective forces, namely density dependent M3Y [11] interactions and the Gogny D1S force. We show in Fig. 3 com- 

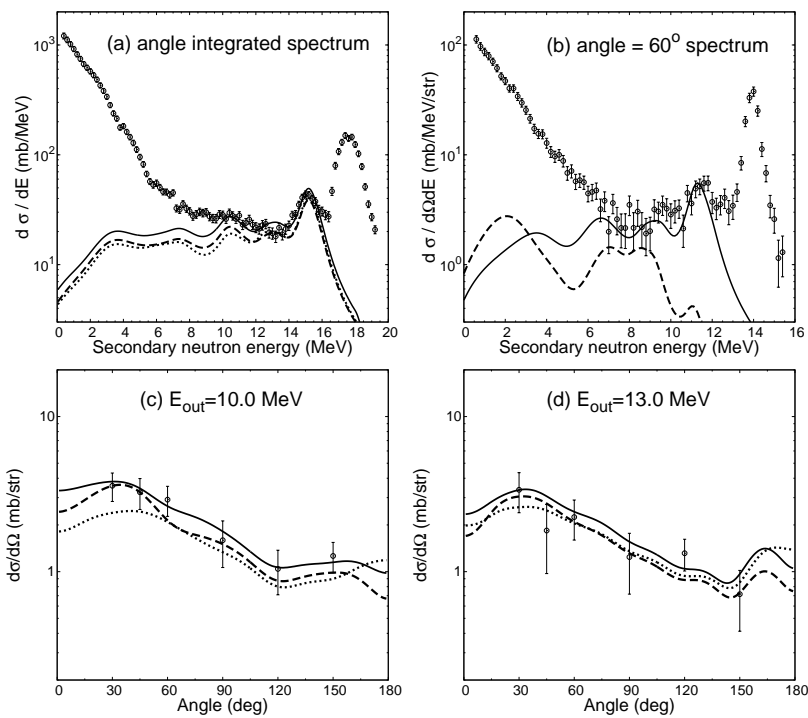

Fig. 3. Comparison between experimental data [12] (open circles) and one-step calculations of double-differential cross sections for $18 \mathrm{MeV}$ (a,c,d) and $14.5 \mathrm{MeV}$ (b) neutron scattering off ${ }^{90} \mathrm{Zr}$. (a) angle integrated neutron spectrum, (b) neutron spectrum at $60^{\circ}$, (c) and (d) angular distributions at secondary energies 10 and $13 \mathrm{MeV}$, respectively. The different curves are defined in the text.

parisons of the calculated double-differential cross sections with experimental data [12] for neutron scattering off ${ }^{90} \mathrm{Zr}$. Results obtained with the CDM3Y3 (full curves), Melbourne (dashed curves) and D1S (dotted curves) interactions are compared in Figs.3(a,c,d) for $18 \mathrm{MeV}$ incident neutrons. The main difference among these calculations is for the magnitude of both spectrum and angular distributions, while the angular shapes remain similar. This comparison provides us a measure of the uncertainty on predictions as due to the approximate knowledge of residual interactions. This uncertainty could be as large as 25\%. Next, for 14.5 MeV neutrons, we show in Fig.3(b) a comparison between double-differential cross sections for which transition amplitudes are obtained using either RPA (full curve) or particle-hole (dashed curve) wave functions. There, the CDM3Y3 interaction was used as residual interaction for $\hat{V}_{\text {eff }}$, Eq.(1). Calculations with particle-hole excitations are significantly lower than those obtained with RPA solutions for an energy transfer up to $11 \mathrm{MeV}$. Like observed for the $120 \mathrm{MeV}$ proton scattering analysis, this difference illustrates effects of collectivity contents in the ${ }^{90} \mathrm{Zr}$ spectrum. In general, the calculated cross sections (full curves in Fig.3) are in qualitative agreement with the experimental data for both emission spectra and angular distributions. The $25 \%$ uncertainty estimation discussed above does not close this specific discussion because we are also well aware of that these calculations have been performed using the ansatz of local potentials for the distorted waves, which ignores non-locality effects.

\section{One-step direct preequilibrium emission from deformed targets}

The structure properties of axially deformed nuclei are defined as follows. In the intrinsic system of coordinates, the axially deformed single particle (s.p.) levels (i.e. Nilsson levels) are calculated in the $\mathrm{HF}+\mathrm{BCS}$ scheme implemented with the Skyrme force $\mathrm{SkM}^{*}$ [19]. Any s.p. state $\left|k \Omega_{k}\right\rangle$ is calculated as

$$
\left|k \Omega_{k}\right\rangle=\sum_{n_{z} \geq 0, n_{\perp} \geq 0} \sum_{\Sigma= \pm \frac{1}{2}} c_{n_{z} n_{\perp} \Lambda \Sigma}^{(k)}\left|n_{z} n_{\perp} \Lambda \Sigma\right\rangle,
$$

where the set $\left\{n_{z} n_{\perp} \Lambda \Sigma\right\}$ is for quantum numbers of harmonic oscillator (HO) basis states $\left|n_{z} n_{\perp} \Lambda \Sigma\right\rangle$ in cylindrical coordinates. The $\left|k \Omega_{k}\right\rangle$ levels are eigen functions of the total angular momentum projection on the symmetry axis. In this representation, particle-hole excitations are built as

$$
|p h\rangle=v_{p}^{2} v_{h}^{2} a_{p}^{+} a_{h}\left|\varphi_{0}\right\rangle,\left|\varphi_{0}\right\rangle=\prod_{k<k_{f}} a_{k}^{+}|-\rangle,
$$

where $v_{p}^{2}$ and $v_{h}^{2}$ are occupancies for particle $(p)$ and hole (h) states, respectively, and where $a_{p}^{+}$and $a_{h}$ are creation and annihilation operators, respectively, acting on the $\mathrm{HF}$ ground state $\left|\varphi_{0}\right\rangle$. Next, the deformed HO basis is transformed into a spherical $\mathrm{HO}$ basis to express the ket $\left|k \Omega_{k}\right\rangle$ into a spherical representation, that is

$$
\left|k \Omega_{k}\right\rangle=\sum_{l \geq 0} \sum_{j=l-\frac{1}{2}}^{j=l+\frac{1}{2}} C_{l j}^{(k)}\left|k l j \Omega_{k}\right\rangle,
$$

where we have introduced the spherical components $\left|k l j \Omega_{k}\right\rangle=b_{k l j \Omega_{k}}^{+}|-\rangle$of the s.p. state $\left|k \Omega_{k}\right\rangle$. More details can be found in [16]. Finally, for our preequilibrium calculations, we adopt the following approximation for physical states

$$
|n J M \Pi\rangle \sim \frac{2 v_{p}^{2} v_{h}^{2} C_{l_{p} j_{p} \Omega_{p}}^{(p)} C_{l_{h} j_{h} \Omega_{h}}^{(h)}}{\sqrt{\left(2 j_{p}+1\right)\left(2 j_{h}+1\right)}}\left[b_{p l_{p} j_{p}}^{+} \otimes b_{h l_{h} j_{h}}^{+}\right]_{M}^{J \Pi}|0\rangle .
$$

This approximation is crude in that the projection on angular momentum is not handled properly. Another approximation we have resorted to consisted in taking local spherical potentials for calculating incoming and outgoing distorted waves.

We display in Fig.4 a comparison between experimental data $[17,18]$ and the double-differential cross sections calculated at the outgoing angles $\Theta=30^{\circ}$ and $90^{\circ}$ for 14.1 MeV and $18 \mathrm{MeV}$ neutrons incident on ${ }^{238} \mathrm{U}$ and ${ }^{232} \mathrm{Th}$, respectively. These calculations (full curves) were performed with CDM3Y3 as the effective interaction $\hat{V}_{\text {eff }}$, Eq.(1). The other curves (defined by various symbols in the plots) represent the contributions of elastic scattering, multistep compound (MSC), evaporation and fission processes (see [20]) for more details), and the sum of all contributions. For ${ }^{238} \mathrm{U}$ our calculations ${ }^{1}$ reproduce the data well

1 An inaccurate theoretical normalization was used in [21] which explains why the present one-step direct calculated values are lower in magnitude than reported previously. 
for $\Theta=30^{\circ}$, (a), and $90^{\circ}$, (c), and for an emission energy up to $\sim 9 \mathrm{MeV}$. These calculations underestimate the experimental cross sections at higher emission energy, that is for an excitation energy in the target in the range 0 to $\sim 5$ $\mathrm{MeV}$. The analysis for the $18 \mathrm{MeV}$ neutron scattering from ${ }^{232} \mathrm{Th}$ displayed in Figs.4(b,d) leads to similar conclusions. In that case data are underestimated above $\sim 13 \mathrm{MeV}$, that is for an excitation energy in the range 0 to $5 \mathrm{MeV}$.

Understanding why calculated cross sections are low in the $0-5 \mathrm{MeV}$ excitation energy range is a long-standing issue that so far has not been fixed in a satisfactory manner. For instance, in a more phenomenological calculation which also used particle-hole excitations, Kawano et al [20] already predicted cross sections which underestimated the ${ }^{238} \mathrm{U}$ data for excitations in the target below $5 \mathrm{MeV}$. Ad hoc collective states, which are not described in the simple particle-hole excitation picture, were introduced as candidates to explain the missing cross section [22]. A different microscopic approach was used in [23,24] where QRPA response functions were used to generate the one-step cross sections. However, this calculation was performed with phenomenological ingredients, namely an adjusted deformed Wood-Saxon potential which defined the s.p. level scheme, and a separable residual interaction with a coupling constant treated as an empirical parameter.

As the issue is related to that of missing collective strengths which have not been identified (typically above $1.4 \mathrm{MeV}$ ) in spectroscopy measurements, we have decided to rely upon a microscopic structure model, namely QRPA as recently implemented with the Gogny force [26]. With this theory, we expect to learn about collectivity of $2^{+}, 3^{-}$ and $4^{+}$levels up to $10 \mathrm{MeV}$ of excitation energy. This is especially relevant to the $K \Pi=3^{-}$octupole strength which so far has escaped experimental identification. Such QRPA calculation may also shed some light as to whether this strength is highly fragmented. Preliminary results for ${ }^{238} \mathrm{U}$ have been obtained with QRPA/D1S calculations in a two quasi-particles space truncated at $65 \mathrm{MeV}$. We display in Fig. 5 the calculated reduced charge transition probabilities for quadrupole and octupole excitations. Note that calculations with no truncation [27] are under progress to obtain fully converged values but the present results already provide good estimates for the response functions. As seen in Fig.5, the octupole response function is mainly concentrated below $6 \mathrm{MeV}$. The quadrupole response function displays large components above $10 \mathrm{MeV}$ but some strength is also concentrated below $5 \mathrm{MeV}$. These components of the response functions are expected to lead to large calculated neutron cross sections wherever needed to improve our description of the spectra showed in Fig.4.

\section{Conclusions}

Summarizing, fully microscopic parameter-free models were used to study medium energy (10-200 MeV) nucleon induced reactions.

Differential cross sections for inelastic scattering of intermediate energy protons off singly- and doubly-closedshell nuclei have been predicted for excited states of di-
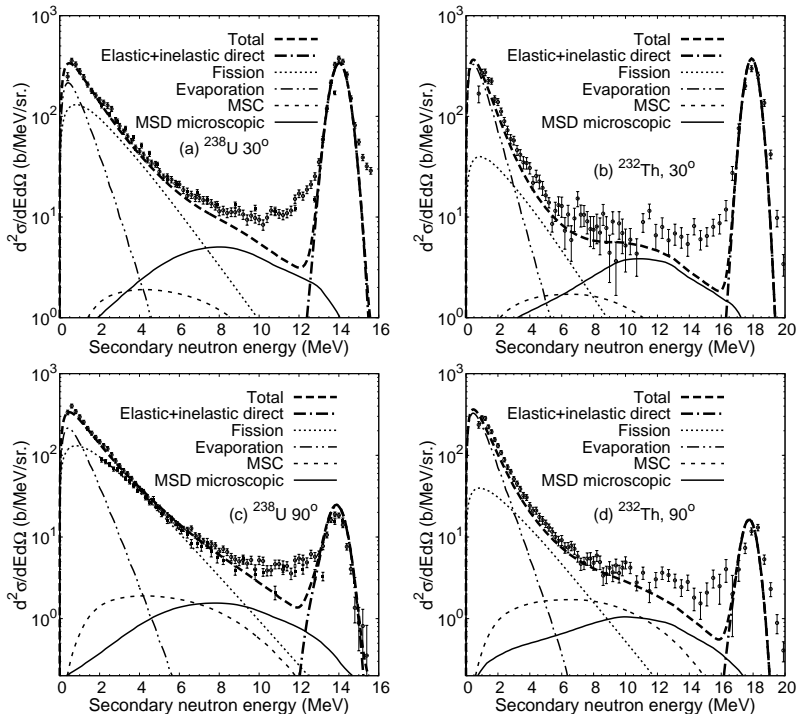

Fig. 4. Comparison between experimental data $[17,18]$ (black circles) and one-step contributions (full curves) to the doubledifferential cross sections for $14.1 \mathrm{MeV}$ neutron on ${ }^{238} \mathrm{U}(\mathrm{a}, \mathrm{c})$ and $18 \mathrm{MeV}$ neutron on ${ }^{232} \mathrm{Th}(\mathrm{b}, \mathrm{d})$. Outgoing angles are indicated on the plots. The various dashed and dotted curves are defined on the plots and in the text.
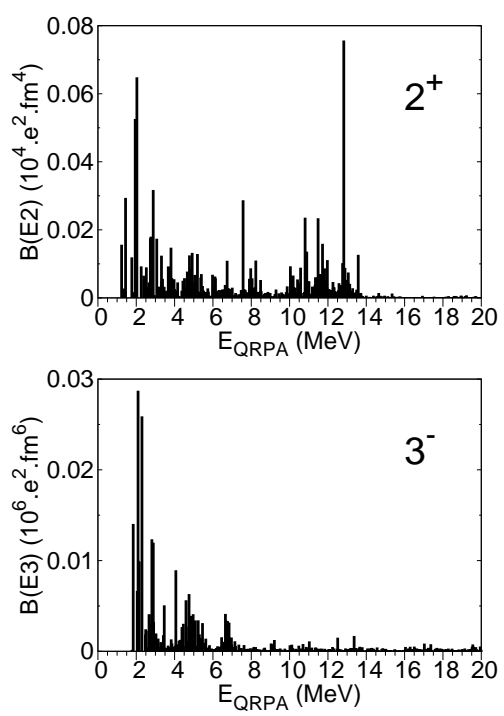

Fig. 5. Reduced charge transition probabilities $\mathrm{B}(\mathrm{E} 2) \uparrow$ (top) and $\mathrm{B}(\mathrm{E} 3) \uparrow$ (bottom) in ${ }^{238} \mathrm{U}$ obtained in QRPA calculations with D1S.

verse nature. The Melbourne g-matrix was used as the effective in-medium NN interaction, and the density matrices required in the scattering calculations (elastic and inelastic) were obtained from RPA model calculations made using the D1S Gogny force. Excellent agreement with ${ }^{208} \mathrm{~Pb}$ cross section data has been obtained for proton inelastic scattering leading to different final states and for a number of incident energies from 54 to $201 \mathrm{MeV}$. Very good predictions were also obtained for the $3{ }_{1}^{-}$excitations in other targets. 
This model was extended beyond discrete excitations, into the continuum, to analyze the preequilibrium emission associated with incoming nucleons. The Melbourne g-matrix and the RPA/D1S theory were also used to calculate all the transition amplitudes for the first order of the preequilibrium MSD process. The one-step cross sections were calculated for intermediate energy proton scattering off ${ }^{90} \mathrm{Zr}$ and our predictions reproduce the data very well. This goal has been achieved only with microscopic ingredients, and no phenomenological input or arbitrary renormalization process enters our analyzes. We have extended this preequilibrium calculation at lower energy to study neutron induced reactions below $20 \mathrm{MeV}$ on ${ }^{90} \mathrm{Zr}$. Other effective interactions than the Melbourne g-matrix were used in this analysis. The predicted neutron spectra and angular distributions are in rather good agreement with the data. The approximate knowledge of some of the model ingredients leads to uncertainties on the absolute normalization of the predicted cross sections. An improvement could be introducing an accurate microscopic optical potential for neutron below $20 \mathrm{MeV}$ instead of a local phenomenological potential. Such potentials are being built [9] to correct the g-matrix at low energy. In proton and neutron scattering off ${ }^{90} \mathrm{Zr}$ analyzes, a comparison between calculations performed with RPA excitations and with incoherent particlehole excitations of the HF mean field demonstrates that a good description of the collectivity content of the target nucleus is crucial to achieve a good understanding of the one-step direct preequilibrium emission.

We have also performed a microscopic calculation of the one-step direct cross sections for neutron scattering off ${ }^{238} \mathrm{U}$ and ${ }^{232} \mathrm{Th}$ below $20 \mathrm{MeV}$. In that case, the spectrum of the target is described in an approximate way by incoherent particle-hole excitation of the deformed HartreeFock + BCS mean field. The results are in rather good agreement with the experimental data for an excitation in the target above $\sim 5 \mathrm{MeV}$. However our calculations underestimate the data at high emission energy. The missing cross section probably arises from excitation of low energy vibrational states which are not described with incoherent particle-hole excitations. New self-consistent QRPA/D1S calculations may help curing this discrepancy. A direct extension of our model will be to take into account all the excitations and transition strengths predicted by the QRPA method.

Authors warmly thank D. Gogny for advice and critical discussion.

\section{References}

1. H. Feshbach, A. Kerman, and A.K. Koonin, Ann. Phys. (N.Y.) 125, 429 (1980).

2. J.F. Berger, M. Girod, and D. Gogny, Comput. Phys. Commun. 159, 365 (1991).

3. M. Dupuis, S. Karataglidis, E. Bauge, J.P. Delaroche, and D. Gogny, Phys. Rev. C 73, 014605 (2006).

4. K. Amos, P.J. Dortmans, H.V. von Geramb, S. Karataglidis, and J. Raynal, Adv. Nucl. Phys. 25, 275 (2000).
5. J.-P. Blaizot and D. Gogny, Nucl. Phys. A284 429 (1977).

6. M. Dupuis, S. Karataglidis, E. Bauge, J.P. Delaroche, and D. Gogny, Phys. Let. B 665,152 (2008).

7. R. Bonetti, A.J Koning, J.M. Akkermans, and P.E. Hodgson, Phys. Rep. 247,1 (1994).

8. A.A. Cowley et al, Phys. Rev. C43, 678 (1991).

9. G. Blanchon, M. Dupuis, N. Vinh Mau, and E. Bauge, see contribution in the present proceedings.

10. A.J. Koning, and J.P. Delaroche, Nucl. Phys. A713, 231 (2003).

11. D.T. Khoa, G.R. Satchler, and W. von Oertzen, Phys. Rev. C 56, 954 (1997).

12. M. Baba, M. Ishikawa, N. Yabuta, T. Kikuchi, H. Wakabayashi, and N. Hirakawa in Proc. Int. Conf. on Nuclear Data for Science and Technology, Mito, 30 May-3 June 1988, edited by S. Igarasi, p.1185.

13. M. Dupuis and T. Kawano, Fully microscopic calculation of the one-step direct process for fast neutron induced reactions, submitted for publication in Phys. Rev. C.

14. D. Vautherin, Phys. Rev. C 7, 296 (1973).

15. H. Flocard, P. Quentin, A. Kerman, and D. Vautherin, Nucl. Phys. A203, 443 (1973).

16. L. Bonneau, T. Kawano, T. Watanabe, and S. Chiba, Phys. Rev. C 75, 054618 (2007).

17. M. Baba, H. Wakabayashi, N. Ito, K. Maeda, and N. Irakawa, J. Nucl. Sci. Technol. 27, 601 (1990).

18. M. Baba, S. Matsuyama, N. Ito, K. Maeda, and N. Irakawa, in proceedings of International Conference on Nuclear Data for Science and Technology, Jülich, Germany, 1991, edited by S. M. Qaim (Springer-Verlag, Berlin/Heidelberg, 1992), p.349.

19. J. Bartel, P. Quentin, M. Brack, C. Guet, and H.B. Hakansoon, Nucl. Phys. A386, 79 (1982).

20. T. Kawano, T. Ohsawa, M. Baba, and T. Nakagawa, Phys. Rev. C, 63, 034601 (2001).

21. M. Dupuis, L. Bonneau, and T. Kawano in Int. Workshop on Compound Nuclear reactions and related topics., (2007).

22. M. Chadwick et al, Nuclear Data Sheets 107, 2931 (2006).

23. M. Herman et al, Nuclear Data Sheets 108, 2655 (2007).

24. H. Wienke, R. Capote, M. Herman, and M. Sin, Phys. Rev. C 78, 064611 (2008).

25. S. Péru, H. Goutte, and J.F. Berger, Nucl. Phys. A 788, 44c (2007).

26. S. Péru and H. Goutte, Phys. Rev. C 78, 024305 (2008).

27. S. Péru, G. Gosselin, J.C. Devaux, and M. Dupuis, in preparation. 